\title{
Weak Type Inequality for the Square Function of a Nonnegative Submartingale
}

by

\section{Adam OSĘKOWSKI}

\author{
Presented by Stanisław KWAPIEŃ
}

Summary. Let $f$ be a nonnegative submartingale and $S(f)$ denote its square function. We show that for any $\lambda>0$,

$$
\lambda \mathbb{P}(S(f) \geq \lambda) \leq \frac{\pi}{2}\|f\|_{1},
$$

and the constant $\pi / 2$ is the best possible. The inequality is strict provided $\|f\|_{1} \neq 0$.

1. Introduction. Let $(\Omega, \mathcal{F}, \mathbb{P})$ be a probability space, filtered by $\left(\mathcal{F}_{n}\right)_{n=0}^{\infty}$, a nondecreasing sequence of sub- $\sigma$-algebras of $\mathcal{F}$. Assume $f=$ $\left(f_{n}\right)_{n=0}^{\infty}$ is an adapted sequence of integrable real-valued random variables. The difference sequence $d f=\left(d f_{n}\right)_{n=0}^{\infty}$ of $f$ is given by the equations $d f_{0}=f_{0}$ and $d f_{n}=f_{n}-f_{n-1}, n=1,2, \ldots$. We define the square function of $f$ by

$$
S(f)=\left(\sum_{k=0}^{\infty}\left|d f_{k}\right|^{2}\right)^{1 / 2} .
$$

We will also use the notation

$$
S_{n}(f)=\left(\sum_{k=0}^{n}\left|d f_{k}\right|^{2}\right)^{1 / 2}
$$

and write $\|f\|_{p}=\sup _{n}\left\|f_{n}\right\|_{p}$ for $p \geq 1$.

In the present paper we deal with weak type inequalities for the square function. As shown by Burkholder [2], if $f$ is a martingale or nonnegative submartingale, then

$$
\lambda \mathbb{P}(S(f) \geq \lambda) \leq 3\|f\|_{1}
$$

2000 Mathematics Subject Classification: Primary 60G42; Secondary 60G48. Key words and phrases: submartingale, square function, weak type inequality. 
Cox [5] showed that the best constant in the above inequality for real-valued martingales $f$ equals $\sqrt{e}$ (it is worth mentioning that in the earlier paper [1] Bollobás conjectures that this is the right choice). The purpose of this note is to determine the optimal constant in (1.1) under the assumption that $f$ is a nonnegative submartingale.

THEOREM 1. If $f$ is a nonnegative submartingale, then for any $\lambda>0$,

$$
\lambda \mathbb{P}(S(f) \geq \lambda) \leq \frac{\pi}{2}\|f\|_{1},
$$

and the constant $\pi / 2$ is the best possible. Furthermore, the inequality is strict unless $\|f\|_{1}=0$.

A few words about the organization of the paper. The proof of the inequality (1.2) is based on Burkholder's method, which translates the problem of proving a given (sub-)martingale inequality to the problem of finding a certain special function (for the description of the method, see e.g. [4] or [6]). We construct the function and thus establish (1.2) in Section 2. In the last section we show that the constant $\pi / 2$ cannot be replaced by a smaller one and that (1.2) is strict in all nontrivial cases.

2. The proof of the inequality (1.2). Let us start with the following auxiliary result.

Lemma 1. For any $x \in(0,1)$ and $d>-x$ such that $(x+d)^{2}+d^{2}<1$ we have

$$
\frac{\sqrt{1-x^{2}}-\sqrt{1-(x+d)^{2}-d^{2}}}{x+d}+\arcsin x-\arcsin \frac{x+d}{\sqrt{1-d^{2}}} \leq 0 .
$$

Proof. Denote the left hand side of $(2.1)$ by $F(x, d)$. If we fix $d$ and differentiate with respect to $x$, we obtain

$$
\begin{aligned}
F_{x}(x, d)(x+d)^{2} & =\sqrt{1-(x+d)^{2}-d^{2}}-\sqrt{1-x^{2}}+\frac{d(x+d)}{\sqrt{1-x^{2}}} \\
& =\sqrt{1-x^{2}-2 d(x+d)}-\sqrt{1-x^{2}}-\frac{-2 d(x+d)}{2 \sqrt{1-x^{2}}},
\end{aligned}
$$

which is nonnegative, due to the concavity of the function $t \mapsto \sqrt{t}$. Therefore the inequality $F(x, d) \leq 0$ will be established once we have shown that $F(-d+, d)<0$ for $d<0$ and $F(0+, d) \leq 0$ for $d \geq 0$. Suppose first that $d<0$. Then

$$
F(-d+, d)=\frac{d}{\sqrt{1-d^{2}}}+\arcsin (-d)=\int_{0}^{-d}\left(\frac{1}{\sqrt{1-s^{2}}}-\frac{1}{\sqrt{1-d^{2}}}\right) d s<0 .
$$


If $d=0$, then $F(x, d)=0$ for any $x$. Finally, if $d>0$, then

$$
\begin{aligned}
F(0+, d) & =\frac{1-\sqrt{1-2 d^{2}}}{d}-\arcsin \frac{d}{\sqrt{1-d^{2}}} \\
& =\int_{0}^{d} \frac{\sqrt{1-2 s^{2}}-1}{\left(1-s^{2}\right)\left(1+\sqrt{1-2 s^{2}}\right)} d s<0 .
\end{aligned}
$$

The proof is complete.

The crucial role in this paper is played by the functions $U, V:[0, \infty) \times$ $[0, \infty) \rightarrow \mathbb{R}$, given by

$$
U(x, y)= \begin{cases}1-\sqrt{1-x^{2}-y^{2}}-x \arcsin \frac{x}{\sqrt{1-y^{2}}} & \text { if } x^{2}+y^{2}<1, \\ 1-\pi x / 2 & \text { if } x^{2}+y^{2} \geq 1,\end{cases}
$$

and $V(x, y)=I_{\{y \geq 1\}}-\pi x / 2$.

The key properties of these functions are listed in the lemma below.

Lemma 2. The functions $U, V$ have the following properties.

(i) $U$ is of class $C^{1}$ on $(0, \infty) \times(0, \infty)$.

(ii) For any $x, y \geq 0$, we have

$$
U_{x}(x, y) \leq 0
$$

(if $x=0$, then we understand $U_{x}(0, y)$ as the limit $U_{x}(0+, y)$ ).

(iii) For any $x, y \geq 0$,

and

$$
U(x, y) \geq V(x, y)
$$

$U(x, y) \leq 1-\pi x / 2$.

(iv) For any $x, y \geq 0$ and $d \geq-x$ we have

$$
U\left(x+d, \sqrt{y^{2}+d^{2}}\right) \leq U(x, y)+U_{x}(x, y) d
$$

(again, if $x=0$, then the partial derivative is understood as the limit).

(v) For any $x \geq 0$,

$$
U(x, x) \leq 0 .
$$

Furthermore, the inequality is strict if $x>0$.

Proof. (i) A direct computation shows that

$$
U_{x}(x, y)= \begin{cases}-\arcsin \frac{x}{\sqrt{1-y^{2}}} & \text { if } x^{2}+y^{2}<1 \\ -\pi / 2 & \text { if } x^{2}+y^{2} \geq 1\end{cases}
$$


and

$$
U_{y}(x, y)= \begin{cases}\frac{y \sqrt{1-x^{2}-y^{2}}}{1-y^{2}} & \text { if } x^{2}+y^{2}<1 \\ 0 & \text { if } x^{2}+y^{2} \geq 1\end{cases}
$$

Now it can be easily verified that both derivatives are continuous on $(0, \infty) \times$ $(0, \infty)$

(ii) This follows immediately from the formula for $U_{x}$ above.

(iii) Clearly, it suffices to show the inequalities on the set $\{(x, y): x, y>0$, $\left.x^{2}+y^{2}<1\right\}$. By $(2.8)$ we have, for $(x, y)$ in this set,

$$
\frac{\partial}{\partial x}\left(U(x, y)+\frac{\pi}{2} x\right)=\frac{\pi}{2}-\arcsin \frac{x}{\sqrt{1-y^{2}}} \geq 0 .
$$

Hence

$$
U(x, y)-V(x, y) \geq U(0, y)-V(0, y)=1-\sqrt{1-y^{2}} \geq 0
$$

and

$$
U(x, y)+\frac{\pi}{2} x \leq U\left(\sqrt{1-y^{2}}, y\right)+\frac{\pi}{2} \sqrt{1-y^{2}}=1 .
$$

(iv) The inequality is easy if $x^{2}+y^{2} \geq 1$ : indeed, we have

$$
U(x, y)+U_{x}(x, y) d=1-\frac{\pi}{2}(x+d) \geq U\left(x+d, \sqrt{y^{2}+d^{2}}\right),
$$

the latter estimate being a consequence of (2.5). Suppose then that $x^{2}+y^{2}$ $<1$. If $(x+d)^{2}+\left(\sqrt{y^{2}+d^{2}}\right)^{2}<1$, then the inequality (2.6) takes the form

$$
\begin{aligned}
&-\sqrt{1-(x+d)^{2}-y^{2}-d^{2}}-(x+d) \arcsin \frac{x+d}{\sqrt{1-y^{2}-d^{2}}} \\
& \leq \sqrt{1-x^{2}-y^{2}}-(x+d) \arcsin \frac{x}{\sqrt{1-y^{2}}} .
\end{aligned}
$$

The first observation is that we may assume that $y=0$ : indeed, if this is not the case, divide both sides by $\sqrt{1-y^{2}}$ and substitute $x:=x / \sqrt{1-y^{2}}$, $d:=d / \sqrt{1-y^{2}}$. The second step is to note that, by continuity, we may assume $x+d>0$. Then the desired estimate is precisely (2.1). The only remaining case is that $x^{2}+y^{2}<1$ and $(x+d)^{2}+\left(\sqrt{y^{2}+d^{2}}\right)^{2} \geq 1$; then the inequality (2.6) is equivalent to

$$
\sqrt{1-x^{2}-y^{2}}+(x+d)\left(\frac{\pi}{2}-\arcsin \frac{x}{\sqrt{1-y^{2}}}\right)-1 \geq 0 .
$$

It is clear that it suffices to prove it for the least possible $d$, i.e., satisfying $d \geq 0$ and $(x+d)^{2}+\left(\sqrt{y^{2}+d^{2}}\right)^{2}=1$. However, then the estimate follows from continuity and the case $x^{2}+y^{2}<1,(x+d)^{2}+\left(\sqrt{y^{2}+d^{2}}\right)^{2}<1$ already considered. 
(v) This is a consequence of (iv): let $x=y=0$ to obtain $U(d, d) \leq$ $U(0,0)+U_{x}(0+, 0) d=U(0,0)=0$. Furthermore, for $d>0$ the inequality is strict: this is precisely $(2.2)$.

Now we are ready to prove the main estimate of the paper.

Proof of (1.2). Let $f$ be any nonnegative submartingale. By homogeneity, it suffices to show (1.2) for $\lambda=1$ only. First we will show that the process $\left(U\left(f_{n}, S_{n}(f)\right)\right)_{n=0}^{\infty}$ is a supermartingale. To this end, fix $n \geq 1$ and observe that, by $(2.6)$,

$$
\begin{aligned}
U\left(f_{n}, S_{n}(f)\right) & =U\left(f_{n-1}+d f_{n}, \sqrt{S_{n-1}(f)+\left|d f_{n}\right|^{2}}\right) \\
& \leq U\left(f_{n-1}, S_{n-1}(f)\right)+U_{x}\left(f_{n-1}, S_{n-1}(f)\right) d f_{n}
\end{aligned}
$$

Both sides are integrable: indeed, one easily checks that $|U(x, y)| \leq K+\pi x / 2$ for some absolute constant $K$; furthermore, $U_{x}(x, y)$ is bounded, in view of (2.8). Therefore, applying the conditional expectation with respect to $\mathcal{F}_{n-1}$ and using (2.3) together with the submartingale property yields

$$
\begin{aligned}
\mathbb{E}\left[U\left(f_{n}, S_{n}(f)\right) \mid \mathcal{F}_{n-1}\right] & \leq U\left(f_{n-1}, S_{n-1}(f)\right)+U_{x}\left(f_{n-1}, S_{n-1}(f)\right) \mathbb{E}\left(d f_{n} \mid \mathcal{F}_{n-1}\right) \\
& \leq U\left(f_{n-1}, S_{n-1}(f)\right) .
\end{aligned}
$$

Combined with (2.4), this will imply the inequality (1.2) for the submartingales $f$ of finite length (that is, satisfying $\mathbb{P}\left(d f_{n}=d f_{n+1}=\cdots=0\right)=1$ for some $n$ ). Namely, for any $n=0,1,2, \ldots$, we write

$$
\begin{aligned}
\mathbb{P}\left(S_{n}(f) \geq 1\right)-\frac{\pi}{2} \mathbb{E} f_{n} & =\mathbb{E} V\left(f_{n}, S_{n}(f)\right) \\
& \leq \mathbb{E} U\left(f_{n}, S_{n}(f)\right) \leq \mathbb{E} U\left(f_{0}, S_{0}(f)\right) \leq 0,
\end{aligned}
$$

where in the last passage we have used the equality $f_{0}=S_{0}(f)$ and the inequality (2.7). The final step is to let $n \rightarrow \infty$ : for any $\varepsilon>0$, we have, by (2.9) applied to the submartingale $f /(1-\varepsilon)$,

$$
\begin{aligned}
\mathbb{P}(S(f) \geq 1) & \leq \lim _{n \rightarrow \infty} \mathbb{P}\left(S_{n}(f) \geq 1-\varepsilon\right) \\
& \leq \lim _{n \rightarrow \infty} \frac{\pi}{2(1-\varepsilon)} \mathbb{E} f_{n} \leq \frac{\pi}{2(1-\varepsilon)}\|f\|_{1} .
\end{aligned}
$$

Now let $\varepsilon \rightarrow 0$ to complete the proof.

\section{Strictness and sharpness}

3.1. Strictness. Suppose $\|f\|_{1}>0$ and observe that if this is the case, then with no loss of generality we may assume that $\mathbb{P}\left(f_{0}>0\right)>0$. Arguing as in (2.9) and (2.10), we obtain

$$
\mathbb{P}(S(f) \geq 1) \leq \frac{\pi}{2}\|f\|_{1}+\mathbb{E} U\left(f_{0}, S_{0}(f)\right) .
$$

It suffices to note that since $f_{0}=S_{0}(f)$ almost surely, it follows that $\mathbb{E} U\left(f_{0}, S_{0}(f)\right)<0$, by Lemma $2(\mathrm{v})$. This yields the claim. 
3.2. Sharpness. Throughout this subsection we assume that the underlying probability space is the interval $[0,1]$ equipped with its Borel subsets and Lebesgue's measure. We will show that the constant is optimal even if we restrict ourselves to the submartingales $f$ satisfying $S(f) \geq 1$ almost surely. One could show this by giving appropriate examples; however, we take the opportunity here to provide a different proof.

Recall that the process $f$ is called simple if it is of finite length (hence its limit $f_{\infty}$ exists almost surely) and for any $n$ the variable $f_{n}$ takes only a finite number of values. For any $(x, y)$, let $Z(x, y)$ be the class of all nonnegative simple submartingales $f$ for which $f_{0}=x$ and $y^{2}-x^{2}+S^{2}(f) \geq 1$ almost surely. Here the filtration is no longer fixed - it may be different for different submartingales.

Lemma 3. Let the function $W:[0, \infty) \times[0, \infty) \rightarrow \mathbb{R}$ be given by

$$
W(x, y)=\inf _{f \in Z(x, y)} \mathbb{E} f_{\infty} .
$$

The function $W$ has the following properties:

(i) For all $x \geq 0, y \in[0,1)$,

$$
W(x, y)=\sqrt{1-y^{2}} W\left(x / \sqrt{1-y^{2}}, 0\right) .
$$

(ii) For all $x, y, d \geq 0$,

$$
W\left(x+d, \sqrt{y^{2}+d^{2}}\right) \geq W(x, y) .
$$

(iii) For all $x, y \geq 0, \alpha \in(0,1)$ and any $d_{1}, d_{2} \geq-x$ satisfying $\alpha d_{1}+$ $(1-\alpha) d_{2}=0$,

$$
\alpha W\left(x+d_{1}, \sqrt{y^{2}+d_{1}^{2}}\right)+(1-\alpha) W\left(x+d_{2}, \sqrt{y^{2}+d_{2}^{2}}\right) \geq W(x, y) .
$$

Proof. (i) Suppose $f$ is a simple nonnegative submartingale. Then $f$ lies in $Z(x, y)$ if and only if $f^{\prime}=f / \sqrt{1-y^{2}}$ belongs to the class $Z\left(x / \sqrt{1-y^{2}}, 0\right)$; indeed, $f_{0}=x$ is equivalent to $f_{0}^{\prime}=x / \sqrt{1-y^{2}}$, and furthermore

$$
y^{2}-x^{2}+S^{2}(f) \geq 1
$$

is equivalent to

$$
-\frac{x^{2}}{1-y^{2}}+S^{2}\left(f^{\prime}\right) \geq 1
$$

This implies

$$
\begin{aligned}
W(x, y)=\inf _{f \in Z(x, y)} \mathbb{E} f_{\infty} & =\inf _{f^{\prime} \in Z\left(x / \sqrt{1-y^{2}}, 0\right)} \mathbb{E} \sqrt{1-y^{2}} f_{\infty}^{\prime} \\
& =\sqrt{1-y^{2}} W\left(x / \sqrt{1-y^{2}}, 0\right) .
\end{aligned}
$$


(ii) Suppose $f \in Z\left(x+d, \sqrt{y^{2}+d^{2}}\right)$ and consider a sequence $f^{\prime}$ such that, with probability $1, f_{0}^{\prime}=x, d f_{1}^{\prime}=d$ and $d f_{n+1}^{\prime}=d f_{n}$ for $n=1,2, \ldots$ Since $d \geq 0, f^{\prime}$ is a simple submartingale (with respect to its natural filtration) and

$$
y^{2}-x^{2}+S^{2}\left(f^{\prime}\right)=y^{2}+d^{2}+\sum_{n=2}^{\infty}\left|d f_{n}^{\prime}\right|^{2}=y^{2}+d^{2}-(x+d)^{2}+S^{2}(f) \geq 1 .
$$

Hence $f^{\prime} \in Z(x, y)$ and since $f_{n}^{\prime}=f_{n-1}$ for $n=1,2, \ldots$, we have

$$
W(x, y) \leq \mathbb{E} f_{\infty}^{\prime}=\mathbb{E} f_{\infty} .
$$

As $f \in Z\left(x+d, \sqrt{y^{2}+d^{2}}\right)$ was arbitrary, (3.2) follows.

(iii) We will use the so-called "splicing" argument; see e.g. [3] for details. Let $f^{(1)}, f^{(2)}$ be two submartingales belonging to $Z\left(x+d_{1}, \sqrt{y^{2}+d_{1}^{2}}\right)$ and $Z\left(x+d_{2}, \sqrt{y^{2}+d_{2}^{2}}\right)$, respectively. Consider the process $f$ such that (recall that $\Omega=[0,1])$

$$
f_{0}=x I_{[0,1]}, \quad d f_{1}=d_{1} I_{[0, \alpha]}+d_{2} I_{(\alpha, 1]}
$$

and, for $\omega \in \Omega$,

$$
d f_{n}(\omega)=d f_{n-1}^{(1)}(\omega / \alpha) I_{[0, \alpha]}(\omega)+d f_{n-1}^{(2)}((\omega-\alpha) /(1-\alpha)) I_{(\alpha, 1]}(\omega)
$$

for $n=2,3, \ldots$. It can be verified easily that $f$ is a simple nonnegative submartingale such that $y^{2}-x^{2}+S^{2}(f)(\omega)$ equals

$$
\begin{aligned}
& {\left[y^{2}+d_{1}^{2}-\left(x+d_{1}\right)^{2}+S^{2}\left(f^{(1)}\right)(\omega / \alpha)\right] I_{[0, \alpha]}(\omega)} \\
& \quad+\left[y^{2}+d_{2}^{2}-\left(x+d_{2}\right)^{2}+S^{2}\left(f^{(2)}\right)((\omega-\alpha) /(1-\alpha))\right] I_{(\alpha, 1]}(\omega) \geq 1 .
\end{aligned}
$$

Thus $f \in Z(x, y)$. Moreover, by the construction, we have

$$
f_{\infty}(\omega)=f_{\infty}^{(1)}(\omega / \alpha)+f_{\infty}^{(2)}((\omega-\alpha) /(1-\alpha)),
$$

so

$$
W(x, y) \leq \mathbb{E} f_{\infty}=\alpha \mathbb{E} f_{\infty}^{(1)}+(1-\alpha) \mathbb{E} f_{\infty}^{(2)},
$$

and since $f^{(1)}, f^{(2)}$ were arbitrary, the inequality (3.3) is satisfied.

The lemma above is the tool to show that $\pi / 2$ in (1.2) is the best possible.

Sharpness of (1.2). In terms of the function $W$, the proof will be complete if we show that $W(0,0) \leq 2 / \pi$. Let $N$ be a fixed (large) integer and $\delta=1 /(N+1)$. By (3.2), applied to $x=y=0$ and $d=\delta$, we have

$$
W(0,0) \leq W(\delta, \delta) .
$$

Now, for $n \in\{1, \ldots, N\}$, use (3.3) with $x=n \delta, y=\sqrt{n} \delta, d_{1}=-n \delta, d_{2}=\delta$ 
and $\alpha=1 /(n+1)$ to obtain

$$
\begin{aligned}
W(n \delta, \sqrt{n} \delta) & \leq \frac{W\left(0, \sqrt{n \delta^{2}+n^{2} \delta^{2}}\right)}{n+1}+\frac{n W((n+1) \delta, \sqrt{n+1} \delta)}{n+1} \\
& =\frac{\sqrt{1-n \delta^{2}-n^{2} \delta^{2}}}{n+1} W(0,0)+\frac{n W((n+1) \delta, \sqrt{n+1} \delta)}{n+1},
\end{aligned}
$$

where in the last passage we have exploited (3.1). This inequality yields

$$
\frac{W(n \delta, \sqrt{n} \delta)}{n}-\frac{W((n+1) \delta, \sqrt{n+1} \delta)}{n+1} \leq \frac{\sqrt{1-n^{2} \delta^{2}}}{n(n+1)} W(0,0),
$$

and combining this with (3.4), we get

$$
W(0,0) \leq \frac{W((N+1) \delta, \sqrt{N+1} \delta)}{N+1}+W(0,0) \sum_{n=1}^{N} \frac{\sqrt{1-n^{2} \delta^{2}}}{n(n+1)} .
$$

Now we make two observations. First, we have $W((N+1) \delta, \sqrt{N+1} \delta)=$ $W(1, \sqrt{\delta})=1$. To see this, note that for any submartingale $f \in Z(1, \sqrt{\delta})$ we have $\mathbb{E} f_{\infty} \geq \mathbb{E} f_{0}=1$, so $W(1, \sqrt{\delta}) \geq 1$. On the other hand, the martingale $f$ starting from 1 such that $d f_{1}=-I_{[0,1 / 2)}+I_{[1 / 2,1]}$ and $d f_{n}=0$ for $n \geq 2$, belongs to $Z(1, \sqrt{\delta})$ and satisfies $\mathbb{E} f_{\infty}=\mathbb{E} f_{0}=1$. The second observation is that

$$
\sum_{n=1}^{N} \frac{1}{n(n+1)}=1-\frac{1}{N+1}
$$

Therefore, (3.5) can be rewritten in the form

$$
W(0,0) \leq 1+W(0,0) \sum_{n=1}^{N} \delta \frac{\sqrt{1-n^{2} \delta^{2}}-1}{n \delta(n+1) \delta} .
$$

Now if we let $N \rightarrow \infty$ (so $\delta \rightarrow 0$ ), then the sum above converges to $\int_{0}^{1}\left(\sqrt{1-x^{2}}-1\right) x^{-2} d x=1-\pi / 2$ and then the inequality becomes $W(0,0) \leq$ $2 / \pi$. This completes the proof.

Acknowledgements. This research was supported by Foundation for Polish Science.

\section{References}

[1] B. Bollobás, Martingale inequalities, Math. Proc. Cambridge Philos. Soc. 87 (1980), 377-382.

[2] D. L. Burkholder, Distribution function inequalities for martingales, Ann. Probab. 1 (1973), 19-42.

[3] - Martingale and Fourier analysis in Banach spaces, in: Probability and Analysis (Varenna, 1985), Lecture Notes in Math. 1206, Springer, 1986, 61-108. 
[4] D. L. Burkholder, The best constant in the Davis inequality for the expectation of the martingale square function, Trans. Amer. Math. Soc. 354 (2002), 91-105.

[5] D. Cox, The best constant in Burkholder's weak- $L^{1}$ inequality for the martingale square function, Proc. Amer. Math. Soc. 85 (1982), 427-433.

[6] A. Osękowski, Two inequalities for the first moment of a martingale, its square and maximal function, Bull. Polish Acad. Sci. Math. 53 (2005), 441-449.

Adam Osękowski

Institute of Mathematics

University of Warsaw

Banacha 2

02-097 Warszawa, Poland

E-mail: ados@mimuw.edu.pl 